SCIENTIFIC LETTER

\title{
Thrombolysis in patients with a brain tumour
}

\section{R Rubinshtein, R Jaffe, M Y Flugelman, B Karkabi, B S Lewis}

$M$ any potentially eligible patients $(30-50 \%)$ with acute ST elevation myocardial infarction (STEMI) do not currently receive thrombolytic treatment, partly because of the presence of a contraindication. ${ }^{1}$ Intracranial haemorrhage (ICH) is a severe complication of thrombolysis, with high morbidity and mortality, and an incidence of approximately $0.75 \%$ in the major thrombolytic trials. ${ }^{2}$ Subgroup analysis of these trials identified advanced age, female sex, use of alteplase (versus streptokinase), and systemic hypertension as risk factors for ICH. ${ }^{2}$ Intracranial tumours (ICT) are considered an absolute contraindication for thrombolysis because of their association with spontaneous ICH, although no such cases have been reported in connection with lytic treatment. We previously reported a patient with meningioma treated with streptokinase for myocardial infarction without neurological sequelae. ${ }^{3}$ We now report two additional cases where in the circumstances of acute STEMI patients failed to disclose the presence of a known stable ICT and were treated with thrombolysis.

\section{CASE DETAILS}

Patient 1 is a 66 year old male who presented with acute anterior STEMI. Alteplase treatment resulted in successful reperfusion. The patient later mentioned a history of known pituitary adenoma for which surgery was planned. Seven days later he underwent angioplasty to the left anterior descending coronary artery for recurrent ischaemia/infarction.

Patient 2 is a 77 year old female with acute extensive anterior STEMI who was treated with streptokinase without signs of reperfusion and subsequently underwent successful angioplasty to the left anterior descending coronary artery. She later acknowledged the existence of an intracranial meningioma.

Computerised tomography confirmed the presence of ICT in both patients, without evidence of bleeding. The size of the pituitary adenoma in patient 1 was $1.8 \times 1.5 \times 1.2 \mathrm{~cm}$ and the size of the meningioma in patient 2 was $0.7 \times 0.8 \times 0.8 \mathrm{~cm}$.

\section{DISCUSSION}

ICTs are found in 1-2\% of all necropsies. ${ }^{4}$ In the GUSTO-1 study, which enrolled 41021 patients, 244 cases of thrombolysis related ICH were systematically investigated with computerised tomography. ${ }^{5}$ Despite the statistical likelihood that approximately $1 \%$ of the participants had ICT, no such cases were reported in conjunction with ICH. It is possible, however, that in the case of a small tumour and a large bleed, the original pathology may have been difficult to identify. We suggest that thrombolysis be considered in selected patients with acute extensive (especially anterior) STEMI and coincidental non-infiltrating ICT, particularly when urgent primary angioplasty is not available.

One should consider the significant mortality reduction achieved with thrombolysis for acute myocardial infarction (up to $50 \%$ ) and the data from the thrombolytic mega-trials that did not identify asymptomatic ICT as a risk factor for ICH. The low ICH rate and the significant benefit of thrombolytic treatment reported in real life patients with STEMI who have contraindications to that therapy might also support our hypothesis. ${ }^{1}$ On the other hand, thrombolysis should probably be avoided in patients with ICT and a small (inferior), low risk STEMI.

\section{Authors' affiliations}

R Rubinshtein, R Jaffe, M Y Flugelman, B Karkabi, B S Lewis, Department of Cardiovascular Medicine, Lady Davis Carmel Medical Center, Haifa, Israel

Correspondence to: Dr Ronen Rubinshtein, Department of Cardiovascular Medicine, Lady Davis Carmel Medical Center, 7 Michal Street, Haifa 34362, Israel; adironen@netvision.net.il

Accepted 16 February 2004

\section{REFERENCES}

1 Ottesen MM, Køber L, Jørgensen S, Torp-Pedersen C, and on behalf of the TRACE Study Group. Consequences of overutilization and underutilization of thrombolytic therapy in clinical practice. J Am Coll Cardiol 2001;37:1581-7.

2 Fibrinolytic Therapy Trailists' (FTT) Collaborative Group. Indications for fibrinolytic therapy in suspected acute myocardial infarction: collaborative overview of early mortality and major morbidity results from all randomized trials of more than 1000 patients. Lancet 1994;343:311-22.

3 Jaffe R, Reichman JM, Weiss AT, et al. Thrombolysis in the presence of an intracranial meningioma. Chest 1997;111:258.

4 Annegers JF, Schoenberg BS, Okazaki H, et al. Epidemiologic study of primary intracranial neoplasms. Arch Neurol 1981;38:217-9.

5 Gebel JM, Sila CA, Sloan MA, et al. Thrombolysis-related intracranial hemorrhage: a radiographic analysis of 244 cases from the GUSTO-1 trial with clinical correlation. Global utilization of streptokinase and tissue plasminogen activator for occluded coronary arteries. Stroke 1998;29:563-9. 